\title{
Salivary duct carcinoma treated with cetuximab-based targeted therapy: A case report
}

\author{
KENTA KAWAHARA $^{1,2}$, AKIMITSU HIRAKI ${ }^{3}$, RYOJI YOSHIDA ${ }^{1}$, HIDETAKA ARITA ${ }^{1}$, \\ YUICHIRO MATSUOKA $^{1}$, TOSHIO YAMASHITA ${ }^{2,3}$, KAN-ICHI KOGA ${ }^{1}$, MASASHI NAGATA ${ }^{1}$, \\ AKIYUKI HIROSUE ${ }^{1}$, DAIKI FUKUMA ${ }^{1}$ and HIDEKI NAKAYAMA ${ }^{1}$ \\ ${ }^{1}$ Department of Oral and Maxillofacial Surgery, Sensory and Motor Organ Sciences, Faculty of Life Sciences, \\ Kumamoto University, Kumamoto 860-8556; ${ }^{2}$ Department of Oral and Maxillofacial Surgery, Amakusa \\ Central General Hospital, Amakusa, Kumamoto 863-0033; ${ }^{3}$ Division of Oral Oncology, Department \\ of Oral and Maxillofacial Surgery, Fukuoka Dental College, Fukuoka 814-0193, Japan
}

Received November 17, 2016; Accepted February 17, 2017

DOI: $10.3892 / \mathrm{mco} .2017 .1226$

\begin{abstract}
Salivary duct carcinoma is a highly aggressive disease with a poor prognosis. Surgical resection is currently the only curative treatment, as there is no effective systemic therapy for this malignancy. Recently, trastuzumab has been shown to exhibit therapeutic efficacy in the treatment of salivary duct carcinoma; similarly, molecularly targeted agents, such as cetuximab, are expected to be useful for salivary duct carcinoma treatment. We herein describe the case of a 56-year-old man diagnosed with salivary duct carcinoma in the left submandibular region, with ipsilateral multiple metastases to the neck lymph nodes. Radical resection of the tumor and submandibular gland with neck dissection were performed. One month after radical surgery, computed tomography (CT) scans indicated metastasis in the lower lobe of the left lung. CT-guided transthoracic fine-needle aspiration biopsy revealed a single metastasis and lung metastasectomy was immediately performed. The tumor cells of the primary lesion and those of the lung metastasis were immunohistochemically positive for epidermal growth factor receptor. One month later, multiple right lung metastases appeared, and the patient was treated with cisplatin/5-fluorouracil (5-FU) chemotherapy
\end{abstract}

Correspondence to: Professor Akimitsu Hiraki, Division of Oral Oncology, Department of Oral and Maxillofacial Surgery, Fukuoka Dental College, 2-15-1 Tamura, Sawara-ku, Fukuoka 814-0193, Japan

E-mail: hiraki@college.fdcnet.ac.jp

Abbreviations: ACC, adenoid cystic carcinoma; AR, androgen receptor; CR, complete response; CT, computed tomography; EGFR, epidermal growth factor receptor; FU, 5-fluorouracil; HER2, human epidermal growth factor receptor 2; S-1, tegafur-gimeracil-oteracil potassium; SD, stable disease; SDC, salivary duct carcinoma; VATS, video-assisted thoracic surgery

Key words: case report, salivary duct carcinoma, cetuximab, neck, lung metastasis, chemotherapy plus cetuximab, achieving a complete radiographic response. However, multiple lung metastases developed during adjuvant weekly cetuximab monotherapy. Subsequently, treatment with S-1 and weekly cetuximab was initiated, and the multiple lung metastases have been maintained as stable disease for 5 months. To the best of our knowledge, this is the first report of cetuximab use for the treatment of salivary duct carcinoma. Although cisplatin/5-FU chemotherapy plus cetuximab was efficacious in treating the lung metastasis, cetuximab monotherapy was insufficient for controlling tumor growth.

\section{Introduction}

Salivary duct carcinoma (SDC) is a rare and highly aggressive type of cancer, accounting for 1-3\% of all malignant salivary tumors (1-4). These lesions typically arise in the parotid gland (75\%), with rare cases (10\%) occurring in the submandibular gland (2). According to the recently revised 2005 World Health Organization classification, SDCs are classified as sarcomatoid, mucin-rich, or invasive micropapillary variants (5). SDC presents as a rapidly growing mass with the potential for local recurrence and cervical and/or distant metastases, and is associated with a high mortality rate. Although surgery, including extended radical resection and ipsilateral neck dissection, followed by postoperative radiation is the standard treatment for SDC $(2,6,7)$, there is currently no standard systemic treatment.

The overall 5-year survival rate associated with SDC is low (42-55\%) (2,7-12). Recent studies have tested molecularly targeted therapies as novel treatment strategies for SDC. This type of cancer resembles intraductal and infiltrating mammary duct carcinomas with a variety of histoarchitectural and cytological patterns. Approximately $61-100 \%$ of the cases are reported to be human epidermal growth factor receptor 2 (HER2)-positive $(13,14)$, and a correlation has been identified between HER 2 expression and prognosis (13-16). Additionally, 50-70\% of SDCs express epidermal growth factor receptor (EGFR; HER1) (17-19), and anti-EGFR-targeted therapies, such as cetuximab, are expected to be useful as novel SDC treatments. Locati et al demonstrated the efficacy of cetuximab 
in 24 of 30 cases of recurrent and/or metastatic salivary gland carcinoma (20). However, the role of molecularly targeted therapies in the treatment of salivary gland carcinomas has not been well defined, and combination therapy comprising cisplatin/5-fluorouracil (5-FU) plus cetuximab has not been tested in SDC. We herein present the first report of clinical activity from systemic treatment comprising cisplatin/5-FU plus cetuximab in a patient with multiple lung metastases from SDC.

\section{Case report}

In August, 2014, a 56-year-old man was referred to the Kumamoto University Hospital due to a gradually increasing swelling in the submandibular region over a 2-month period (Fig. 1A), without pain or paresthesia. The patient's medical history included hypertension and chronic obstructive pulmonary disease. There was no noteworthy familial history of cancer. An extraoral examination revealed a firm lump in the submandibular region, without adhesion to the overlying skin; no neurological disturbance of the lingual or facial nerves was observed. Intraorally, there were no significant findings. Computed tomography (CT) (Fig. 1B) and magnetic resonance imaging (Fig. 1C) revealed a 3.0x3.0-cm heterogeneous mass in the left submandibular region and several necrotic and enlarged lymph nodes in the ipsilateral neck. Positron emission tomography-CT revealed abnormal enhancing lesions with increased fluorodeoxyglucose uptake in the left submandibular gland region and cervical lymph nodes, but no distant metastases (Fig. 1D). Ultrasonography indicated enlarged or necrotic lymph nodes in the submandibular and superior internal jugular region. Therefore, this lesion was classified as cT4N2bM0 according to the World Health Organization International Classification of Tumors (21).

An incisional biopsy was performed under local anesthesia, and the histopathological examination revealed SDC. Thereafter, the submandibular tumor was removed with 1-cm safety margins, followed by radical neck dissection and marginal mandibulectomy. The histopathological examination revealed that all resection margins were tumor-free. The excised lesion primarily comprised cells with large atypical nuclei and prominent nucleoli, and atypical cells with moderately abundant eosinophilic cytoplasm. The tumor contained frequent ductal lesions and central comedonecrosis associated with cribriform and epithelial differentiation. Despite vascular invasion, no nerve invasion was detected. The tumor cells were immunohistochemically positive for EGFR and androgen receptor (AR), but negative for HER2. The patient was ultimately diagnosed with SDC (Fig. 2A).

One month after radical surgery, a CT scan revealed metastasis in the lower lobe of the left lung (Fig. 2B), and CT-guided transthoracic fine-needle aspiration revealed a single metastasis. Lung metastasectomy was performed via video-assisted thoracic surgery (VATS). The lung tumor was histologically diagnosed as metastasis from SDC (Fig. 2C), and the tumor was immunohistochemically positive for EGFR and AR, but negative for HER2 (Fig. 2D-F). One month later, however, another CT scan revealed multiple metastases in the right lung (Fig. 3A), and systemic treatment similar to the regimen used in the EXTREME trial was initiated $(22,23)$. The regimen comprised cetuximab every 7 days (120-min intravenous infusion of a $400-\mathrm{mg} / \mathrm{m}^{2}$ initial dose, followed by weekly 60 -min infusions of $250 \mathrm{mg} / \mathrm{m}^{2}$ ) together with 3-week cycles of cisplatin (240-min intravenous infusion of $80 \mathrm{mg} / \mathrm{m}^{2}$ on day 1) and 5 -FU chemotherapy (24-h continuous infusion of $800 \mathrm{mg} / \mathrm{m}^{2}$ per day on days 1-5) $(22,23)$. This treatment was previously found to be well-tolerated, with mild adverse events, including grade 1 skin rash, dermatitis acneiform and paronychia, and grade 2 hypomagnesemia. All lung metastatic lesions had regressed after two cycles (Fig. 3B), and a complete response (CR) was confirmed after six cycles (Fig. 3C). Although the patient continued to receive cetuximab monotherapy, metastases had developed in both lungs 3 months after the completion of systemic combination therapy. The right lung metastases were removed via VATS, whereas radiofrequency ablation treatment was planned for the left lung due to the extensive multiple metastases. However, as new, rapidly growing metastatic masses appeared (Fig. 4A), the therapeutic strategy was changed to palliative chemotherapy and follow-up. We initiated treatment with 120-mg/day tegafur-gimeracil-oteracil potassium (S-1) daily for 2 weeks, followed by a 1-week rest (24), with concurrent continued weekly cetuximab. After 5 months of treatment, CT scans revealed stable disease (SD) regarding the left lung metastases (Fig. 4B). The patient has been continuing these systemic treatments for 7 months, without any compromise to his quality of life.

The treatment protocol was approved by the Kumamoto University Hospital. Consent to participate in this study was obtained from the patient's family. This investigation was conducted according to the guidelines of the Helsinki Declaration. Written informed consent was obtained from the patient for the publication of this case report and associated images.

\section{Discussion}

SDC was first described in 1968 by Kleinsasser et al (25). This type of cancer is considered to be among the most aggressive salivary gland carcinomas due to the high frequency of local recurrence, as well as associated cervical lymph node and distant metastases (2,7-12). The most common treatment for SDC is surgery, particularly extended radical resection and ipsilateral neck dissection, with postoperative radiotherapy (2,6,7). Between 2005 and 2015, SDC patients were frequently diagnosed at an advanced stage, as in $55-82 \%$ of the cases the disease had already spread to the cervical lymph nodes on first diagnosis. Furthermore, local and regional recurrence and distant metastasis following surgery and postoperative radiotherapy were observed in 11-48, 8-26 and $24-63 \%$ of the patients, respectively, with a 5-year survival rate of $42-55 \%(2,7-12)$.

Although curative postoperative radiotherapy (60-70 Gy) is administered after extended surgery in the majority of the cases, only a few studies have compared surgical resection alone to surgical resection with postoperative radiotherapy. In a previous study involving postoperative radiotherapy, Shinoto et al reported that the local recurrence, regional recurrence, distant metastasis and 5-year survival rates were 28, 16, 48 and 47\%, respectively (7). Despite aggressive treatment with postoperative radiotherapy, treatment efficacy has been 

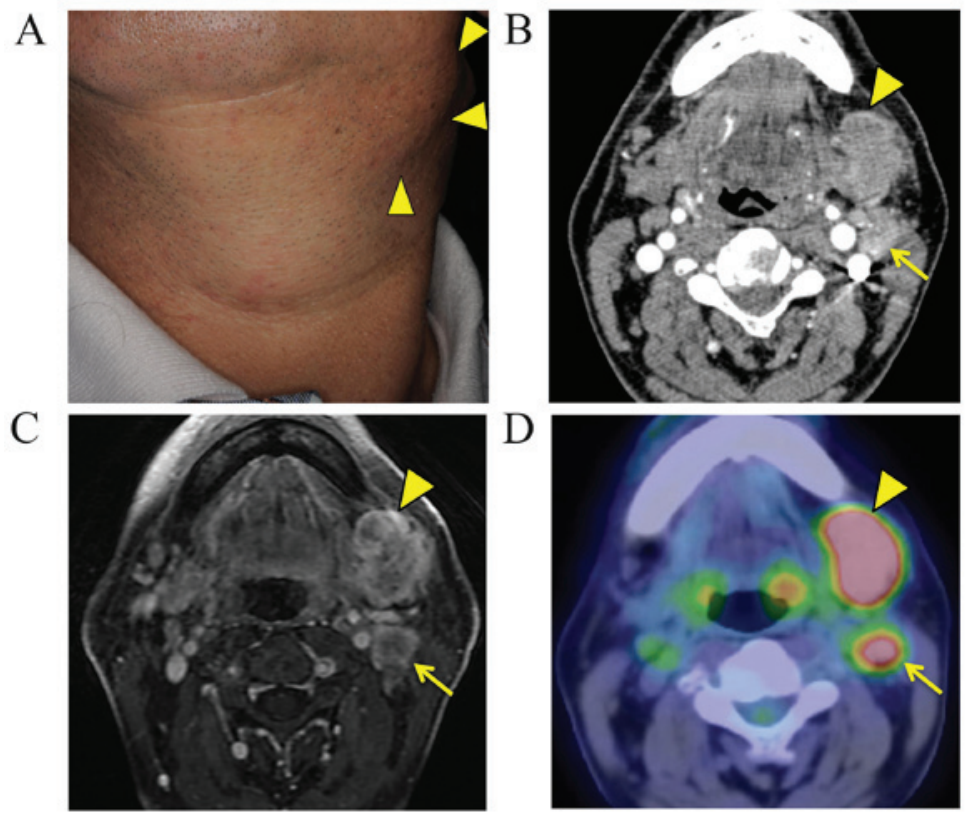

Figure 1. (A) Extraoral image captured at the initial examination, showing a swelling in the submandibular region (arrowheads). (B) Contrast-enhanced computed tomography and (C) contrast-enhanced T1-weighted magnetic resonance images showing the primary tumor (arrowheads) and lymph node metastatic sites (arrows). (D) Pretreatment positron emission tomography-computed tomography image showing the primary tumor (arrowheads) and lymph node metastatic sites (arrows).
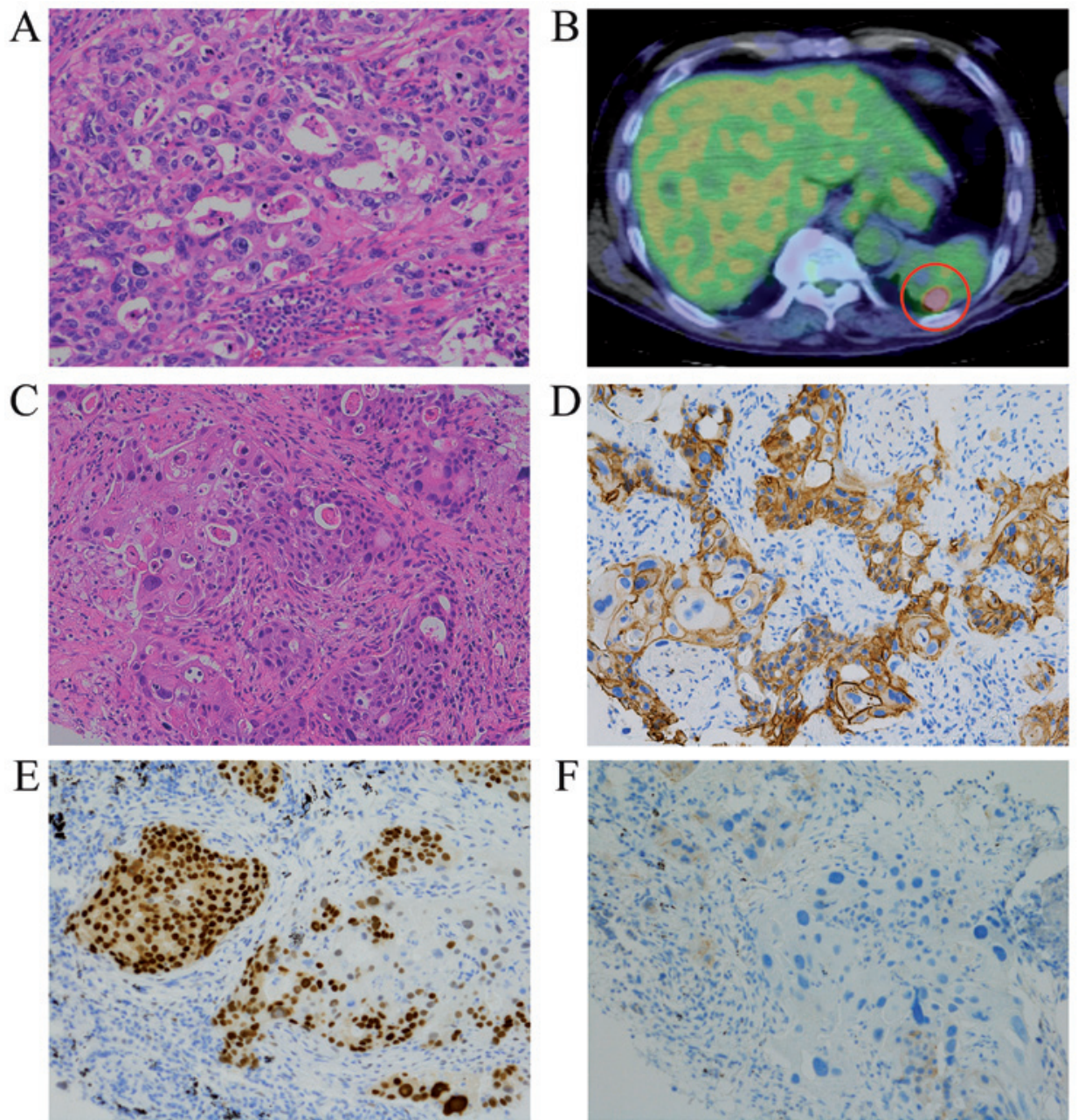

Figure 2. (A) Photomicrograph of the resected primary tumor, stained with hematoxylin and eosin (H\&E). (B) Positron emission tomography-computed tomography image prior to the initial pulmonary metastasectomy. Photomicrographs of the resected lung metastasis including (C) H\&E staining and immunohistochemical staining for (D) epidermal growth factor receptor, (E) androgen receptor and (F) human epidermal growth factor receptor 2. 

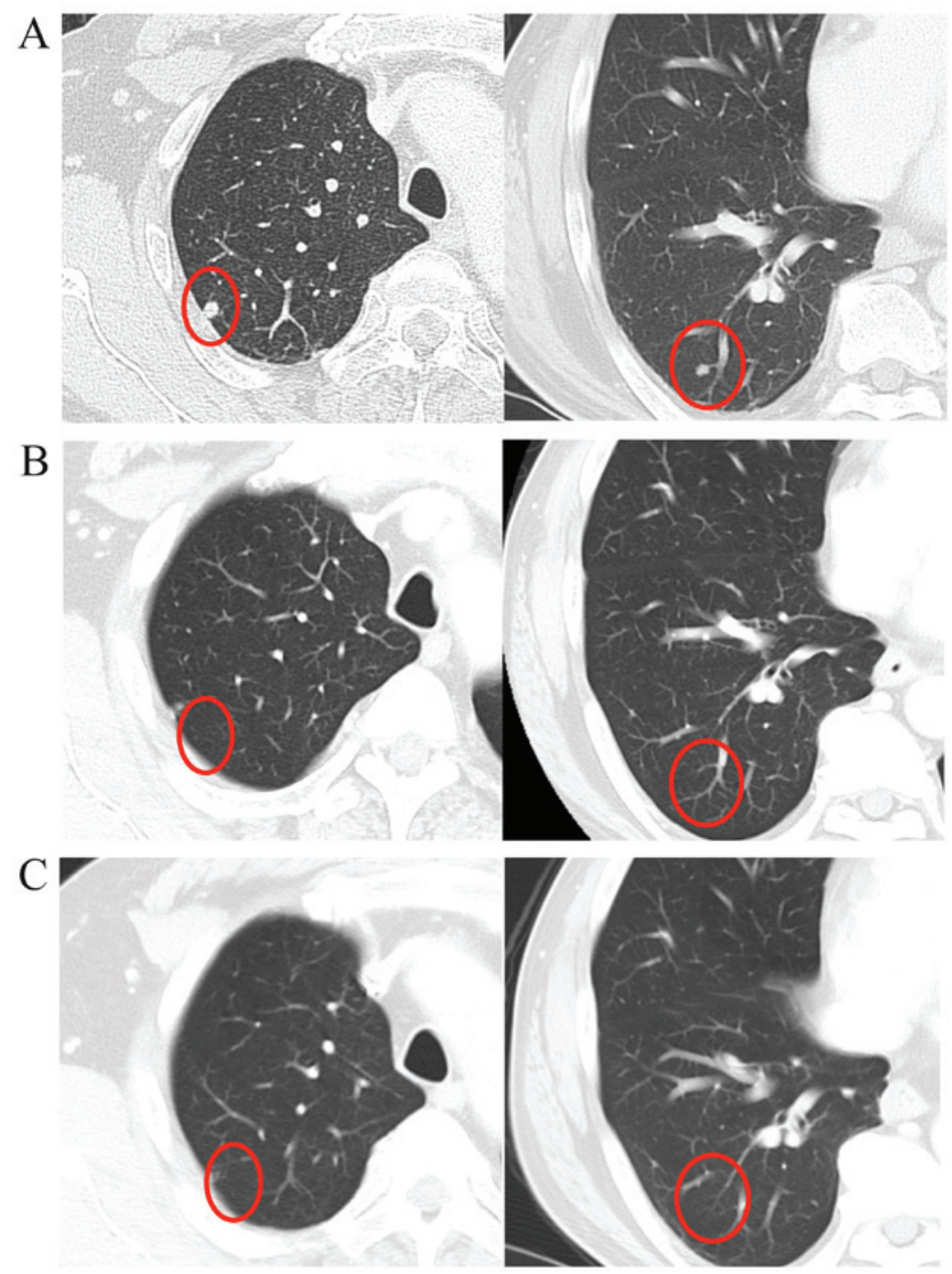

Figure 3. Computed tomography scans showing multiple lung metastases (A) prior to systemic treatment, (B) after two cycles of treatment with cisplatin/5-fluorouracil plus cetuximab and $(\mathrm{C})$ after six cycles of therapy.

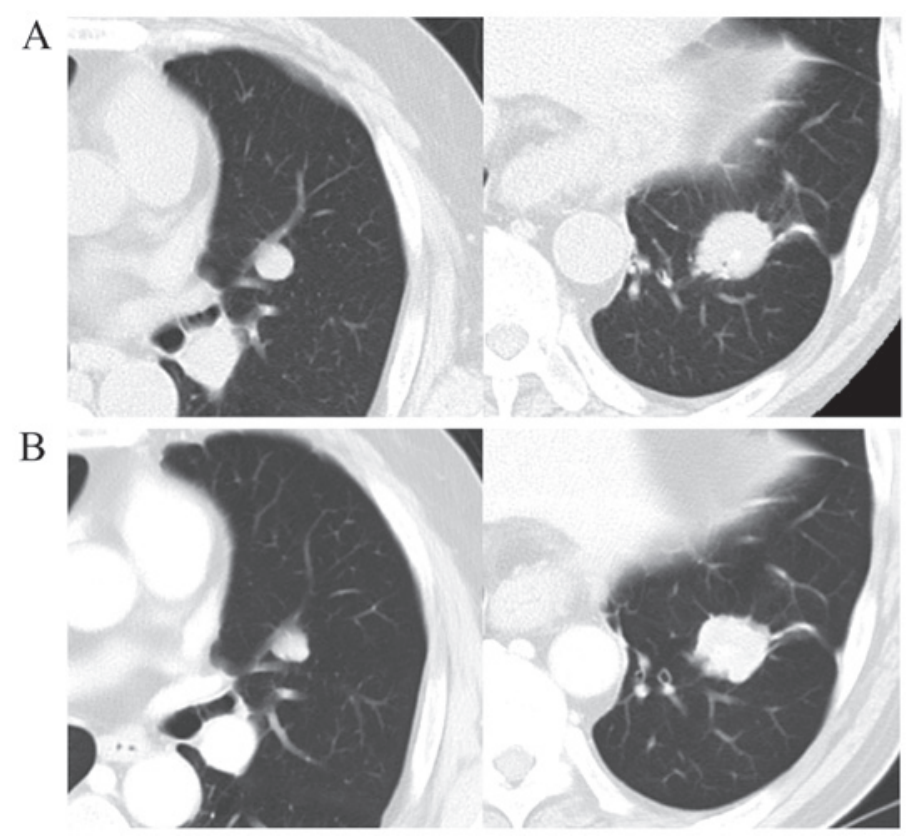

Figure 4. Computed tomography scans showing multiple lung metastases (A) while the patient was on maintenance cetuximab monotherapy and (B) after 2 months of treatment with S-1 and weekly cetuximab. 
insufficient and controversial. In fact, most previous cases involving treatment of local and regional recurrences had poor prognoses. Meredith et al reported distant metastases in 12 of $32(37.5 \%)$ SDC patients with local and regional recurrence. The majority of those patients received palliative therapy or supportive care, although some underwent salvage surgery and additional radiation therapy, alone or combined with chemotherapy. However, all the patients have since succumbed to the disease (9). The most frequent cause of death is distant metastasis, and $65 \%$ of patients with distant metastases succumb to the disease 5 months-10 years later $(5,26,27)$. Although local and regional recurrence are correlated with prognosis, targeting the metastatic potential of SDC is crucial for disease control (28). Sites of distant metastasis include the lungs, bones, liver, skin and brain, although in most cases metastases develop in the lungs and bones $(9,28)$. Systemic chemotherapy should therefore be considered for the treatment of distant metastasis, although there have been no convincing reports regarding chemotherapy for SDC. Chemotherapy comprising platinum-, anthracycline-, or taxane-based regimens has been used, with response rates of $15-50 \%(19,29)$, but no reliable data on the specific chemoresponsiveness of SDC have been published to date (30). Additionally, Meredith et al reported that SDC was not sensitive to chemotherapy (9). Therefore, treatment efficacy remains unclear, and there is currently no consensus regarding chemotherapy for $\operatorname{SDC}(19,31,32)$.

Only a limited number of studies have reported the outcome of lung metastasectomy to remove metastases from head and neck salivary gland carcinoma, particularly from adenoid cystic carcinoma (ACC), whereas none have specifically addressed SDC. Bobbio et al reported that, among 50 patients with ACC lung metastases, the 10 -year survival rates did not differ significantly between patients who did and those who did not undergo complete lung metastasectomy (33). Liu et al reported that, although patients with ACC had a 5-year survival rate of $84 \%$ following metastasectomy, there were no disease-free survivors at 14 years (34), indicating a lack of effective treatments for lung metastasis. ACC spreads in a manner similar to SDC, although metastases of the former tend to develop gradually. By contrast, SDC metastases occur earlier and develop more rapidly, suggesting that the efficacy of lung metastasectomy for SDC may be insufficient when compared to its efficacy for ACC. In the case presented herein, SDC developed in the submandibular gland and the patient had lymph node metastases at initial diagnosis, as described previously (2,7-12). Radical tumor resection with ipsilateral neck dissection were first performed, followed by lung metastasectomy via VATS for left lower lung metastasis and adjuvant chemotherapy. One month after metastasectomy, effective treatments for new multiple right lung metastases were sought, despite the lack of local or regional recurrence.

Recent studies of novel treatment strategies for SDC investigated the systemic use of molecularly targeted therapies (14-16,35-37). Trastuzumab, which targets HER2, effectively prevents the recurrence of metastatic HER2-positive breast cancer. SDC histologically resembles intraductal and infiltrating mammary duct carcinoma, and previous studies reported the therapeutic efficacy of adjuvant or palliative trastuzumab, carboplatin and paclitaxel for SDC, which frequently overexpresses HER2 (14-16,35-37). Limaye et al described 8 patients with HER2-positive SDC who were treated with concurrent radiation and chemotherapy consisting of weekly paclitaxel, carboplatin and trastuzumab after surgical resection, followed by adjuvant trastuzumab monotherapy (16). Of the patients who received adjuvant therapy, $38 \%$ experienced recurrence, but all patients with metastatic disease responded to treatment with trastuzumab. One patient achieved a CR and currently has no evidence of disease (16). Furthermore, trastuzumab was found to be superior to conventional chemotherapy for the treatment of distant metastasis, and palliative and adjuvant trastuzumab-based therapy should be considered for all patients with HER2-positive SDC (14-16,35-37). Despite several reports on the usefulness of trastuzumab, these have mostly been retrospective analyses. Prospective and larger randomized studies with longer follow-up periods are required to confirm the effectiveness of trastuzumab.

Cetuximab, an anti-EGFR monoclonal antibody, is also expected to exhibit therapeutic efficacy. Recently, EGFR inhibitors, such as cetuximab, have increasingly been used to treat salivary gland tumors, which are often resistant to chemotherapy or radiotherapy. Cetuximab-based regimens were used in a phase II study including 30 patients with recurrent or metastatic salivary gland carcinomas, and $>50 \%$ of the treated patients achieved SD (20). Thus, cetuximab is being increasingly recognized as a more effective alternative to conventional chemotherapy. Fan et al reported EGFR expression in $\sim 92 \%$ of SDC cases (17), and a marginally significant correlation of EGFR overexpression in SDC with local recurrence was observed (19). Furthermore, EGFR overexpression in salivary gland tumors was found to be correlated with poor prognosis and appeared to play an important role in tumorigenesis $(38,39)$. Although anti-EGFR targeted therapies are expected to provide novel treatment options, no previous reports have detailed their effectiveness for SDC.

In the present case, radical tumor resection was performed, including marginal mandibulectomy and ipsilateral neck dissection. The pathological examination revealed that all resection margins were tumor-free, and distant metastasis was not detected at the time. Previous reports led us to consider postoperative adjuvant therapy to prevent local and regional recurrence and distant metastasis (2,7-12). Therefore, a systemic molecularly targeted therapy selected for the distant metastasis, along with close follow-up to monitor local and regional recurrence. Unfortunately, 1 month after surgery a left lower lung metastasis was detected, which was treated by VATS-assisted lung metastasectomy. The immunohistochemical analysis revealed that most tumor cells from the primary lymph node metastases and the lung metastasis were immunohistochemically positive for EGFR. Therefore, the planned systemic treatment included cisplatin/5-FU chemotherapy plus cetuximab, as reported in the EXTREME trial (22).

Shortly thereafter, new multiple metastases appeared in the lower lobe of the right lung, and systemic treatment for bilateral lung metastases was promptly initiated. After six cycles of treatment, which was well-tolerated, the patient achieved a CR. These dramatic effects on tumor regression, together with the lack of severe adverse events, suggest that this systemic treatment may lead to improved outcomes for patients with SDC. Although our patient continued to receive 
cetuximab monotherapy in accordance with the EXTREME trial protocol, CT scans revealed right lower, left lower and upper lung metastases. This SDC responded to the combination chemotherapy plus cetuximab, but it did not respond to weekly cetuximab maintenance monotherapy. Although a cisplatin/5-FU regimen would not be expected to exert antitumor effects on SDC, tumor regression was achieved by combining this regimen with cetuximab. Although the underlying mechanism of action has not been fully elucidated, several factors may have contributed to the three-drug combination regimen-mediated control of SDC in our case.

Of note, late-onset cisplatin-induced peripheral neuropathy continuing this three-drug combination regimen difficult. After considering the combination therapy with paclitaxel and cetuximab, cisplatin was replaced with S-1, which may be used to provide long-term and low-impact treatment and maintain the patient's quality of life, according to his wishes. Therefore, S-1 and weekly cetuximab treatment was initiated, and the multiple lung metastases have subsequently been maintained as SD. The effects of this regimen may be similar to those of the initial three-drug combination regimen. Although the results indicate that cisplatin/5-FU chemotherapy plus cetuximab may be an effective treatment for SDC, these results should be interpreted with caution, since this is a case report of a single patient.

We herein presented the first case report of cetuximab-containing chemotherapy for SDC. Even after treatment with extended surgery followed by radiotherapy, $~ 50 \%$ of patients with SDC experience systemic failure, resulting in a poor prognosis. Although the main limitation of our study is that in involves a single case, our findings demonstrated the efficacy of cetuximab-containing chemotherapy as postoperative adjuvant therapy for distant metastases from SDC. Prospective and larger randomized studies with longer follow-up periods are required to confirm our findings.

\section{Acknowledgements}

The present study was supported by the Japan Society for the Promotion of Science KAKENHI, Grant no. 90732735. The authors would like to thank Editage (www.editage.jp) for English language editing.

\section{References}

1. Gal R, Strauss M, Zohar Y and Kessler E: Salivary duct carcinoma of the parotid gland. Cytologic and histopathologic study. Acta Cytol 29: 454-456, 1985

2. Jaehne M, Roeser K, Jaekel T, Schepers JD, Albert N and Löning T: Clinical and immunohistologic typing of salivary duct carcinoma: A report of 50 cases. Cancer 103: 2526-2533, 2005.

3. Seifert G, Batsakis J, Brocheriou C, Cardesa A, Dardick I and Ellis G: World Health Organization. Histological typing of salivary gland tumors. 2nd edition. New York, Springer, 1991.

4. Seifert G and Caselitz J: Epithelial salivary gland tumors: Tumor markers. In: Fenoglio-Preiser CM, Wolff M, Rilke F, editors. Progress in surgical pathology. New York, Field \& Wood, pp157-187, 1989.

5. Barnes L, Eveson J, Reichart P and Sidranksky D: World Health Organization Classification of Tumours. Pathology and genetics of head and neck tumours. Lyon, IARC Press, pp236-237, 2005.

6. McHugh JB, Visscher DW and Barnes EL: Update on selected salivary gland neoplasms. Arch Pathol Lab Med 133: 1763-1774, 2009.
7. Shinoto M, Shioyama Y, Nakamura K, Nakashima T, Kunitake N, Higaki Y, Sasaki T, Ohga S, Yoshitake T, Ohnishi K, et al: Postoperative radiotherapy in patients with salivary duct carcinoma: Clinical outcomes and prognostic factors. J Radiat Res 54: 925-930, 2013.

8. Kim JY, Lee S, Cho KJ, Kim SY, Nam SY, Choi SH, Roh JL, Choi EK, Kim JH, Song SY, et al: Treatment results of post-operative radiotherapy in patients with salivary duct carcinoma of the major salivary glands. Br J Radiol 85: e947-e952, 2012.

9. Johnston ML, Huang SH, Waldron JN, Atenafu EG, Chan K, Cummings BJ, Gilbert RW, Goldstein D, Gullane PJ, Irish JC, et al: Salivary duct carcinoma: Treatment, outcomes, and patterns of failure. Head Neck 8 (Suppl 1): E820-E826, 2016.

10. Salovaara E, Hakala O, Bäck L, Koivunen P, Saarilahti K, Passador-Santos F, Leivo I and Mäkitie AA: Management and outcome of salivary duct carcinoma in major salivary glands. Eur Arch Otorhinolaryngol 270: 281-285, 2013.

11. Weon YC, Park SW, Kim HJ, Jeong HS, Ko YH, Park IS, Kim ST, Baek CH and Son YI: Salivary duct carcinomas: Clinical and $\mathrm{CT}$ and MR imaging features in 20 patients. Neuroradiology 54: 631-640, 2012.

12. Roh JL, Cho KJ, Kwon GY, Choi SH, Nam SY and Kim SY: Prognostic values of pathologic findings and hypoxia markers in 21 patients with salivary duct carcinoma. J Surg Oncol 97: 596-600, 2008.

13. Falchook GS, Lippman SM, Bastida CC and Kurzrock R: Human epidermal receptor 2-amplified salivary duct carcinoma: Regression with dual human epidermal receptor 2 inhibition and anti-vascular endothelial growth factor combination treatment. Head Neck 36: E25-E27, 2014.

14. Xie S, Yang H, Bredell M, Shen S, Yang H, Jin L and Zhang S: Salivary duct carcinoma of the parotid gland: A case report and review of the literature. Oncol Lett 9: 371-374, 2015.

15. Lee JS, Kwon OJ, Park JJ and Seo JH: Salivary duct carcinoma of the parotid gland: Is adjuvant HER-2-targeted therapy required? J Oral Maxillofac Surg 72: 1023-1031, 2014.

16. Limaye SA, Posner MR, Krane JF, Fonfria M, Lorch JH, Dillon DA, Shreenivas AV, Tishler RB and Haddad RI: Trastuzumab for the treatment of salivary duct carcinoma. Oncologist 18: 294-300, 2013.

17. Fan CY, Melhem MF, Hosal AS, Grandis JR and Barnes EL: Expression of androgen receptor, epidermal growth factor receptor, and transforming growth factor alpha in salivary duct carcinoma. Arch Otolaryngol Head Neck Surg 127: 1075-1079, 2001.

18. Williams MD, Roberts D, Blumenschein GR Jr, Temam S, Kies MS, Rosenthal DI, Weber RS and El-Naggar AK: Differential expression of hormonal and growth factor receptors in salivary duct carcinomas: Biologic significance and potential role in therapeutic stratification of patients. Am J Surg Path 31: 1645-1652, 2007.

19. Williams MD, Roberts DB, Kies MS, Mao L, Weber RS and El-Naggar AK: Genetic and expression analysis of HER-2 and EGFR genes in salivary duct carcinoma: Empirical and therapeutic significance. Clin Cancer Res 16: 2266-2274, 2010.

20. Locati LD, Bossi P, Perrone F, Potepan P, Crippa F, Mariani L, Casieri P, Orsenigo M, Losa M, Bergamini C, et al: Cetuximab in recurrent and/or metastatic salivary gland carcinomas: A phase II study. Oral Oncol 45: 574-578, 2009.

21. Seifert G, Brocheriou C, Cardesa A and Eveson JW: WHO International histological classification of tumours. Tentative histological classification of salivary gland tumours. Pathol Res Pract 186: 555-581, 1990.

22. Vermorken JB, Mesia R, Rivera F, Remenar E, Kawecki A, Rottey S, Rottey S, Erfan J, Zabolotnyy D, Kienzer HR, et al: Platinum-based chemotherapy plus cetuximab in head and neck cancer. New Engl J Med 359: 1116-1127, 2008.

23. Guo Y, Shi M, Yang A, Feng J, Zhu X, Choi YJ, Hu G, Pan J, Hu C, Luo R, et al: Platinum-based chemotherapy plus cetuximab first-line for Asian patients with recurrent and/or metastatic squamous cell carcinoma of the head and neck: Results of an open-label, single-arm, multicenter trial. Head Neck 37: 1081-1087, 2015

24. Tsukahara K, Kubota A, Hasegawa Y, Takemura H, Terada T, Taguchi T, Nagahara K, Nakatani H, Yoshino K, Higaki Y, et al: Randomized phase III trial of adjuvant chemotherapy with S-1 after curative treatment in patients with squamous-cell carcinoma of the head and neck (ACTS-HNC). PLoS One 10: $\mathrm{e} 0116965,2015$. 
25. Kleinsasser O, Klein HJ and Hubner G: Salivary duct carcinoma. A group of salivary gland tumors analogous to mammary duct carcinoma. Arch Klin Exp Ohren Nasen Kehlkopfheilkd 192: 100-105, 1968 (In German).

26. Adelstein DJ, Koyfman SA, El-Naggar AK and Hanna EY: Biology and management of salivary gland cancers. Semin Radiat Oncol 22: 245-253, 2012.

27. Barnes L, Rao U, Krause J, Contis L, Schwartz A and Scalamogna P: Salivary duct carcinoma. Part I. A clinicopathologic evaluation and DNA image analysis of 13 cases with review of the literature. Oral Surg Oral Med Oral Pathol 78: 64-73, 1994

28. Guzzo M, Di Palma S, Grandi C and Molinari R: Salivary duct carcinoma: Clinical characteristics and treatment strategies. Head Neck 19: 126-133, 1997.

29. Airoldi M, Fornari G, Pedani F, Marchionatti S, Gabriele P, Succo G and Bumma C: Paclitaxel and carboplatin for recurrent salivary gland malignancies. Anticancer Res 20: 3781-3783, 2000.

30. Surakanti SG and Agulnik M: Salivary gland malignancies: The role for chemotherapy and molecular targeted agents. Semin Oncol 35: 309-319, 2008.

31. Dimery IW, Legha SS, Shirinian M and Hong WK: Fluorouracil, doxorubicin, cyclophosphamide, and cisplatin combination chemotherapy in advanced or recurrent salivary gland carcinoma. J Clin Oncol 8: 1056-1062, 1990.

32. Lagha A, Chraiet N, Ayadi M, Krimi S, Allani B, Rifi H, Raies $\mathrm{H}$ and Mezlini A: Systemic therapy in the management of metastatic or advanced salivary gland cancers. Oral Oncol 48: 948-957, 2012

33. Bobbio A, Copelli C, Ampollini L, Bianchi B, Carbognani P, Bettati S, Sesenna E and Rusca M: Lung metastasis resection of adenoid cystic carcinoma of salivary glands. Eur J Cardiothorac Surg 33: 790-793, 2008
34. Liu D, Labow DM, Dang N, Martini N, Bains M, Burt M, Downey R Jr, Rusch V, Shah J and Ginsberg RJ: Pulmonary metastasectomy for head and neck cancers. Ann Surg Oncol 6: $572-578,1999$.

35. Kaidar-Person O, Billan S and Kuten A: Targeted therapy with trastuzumab for advanced salivary ductal carcinoma: Case report and literature review. Med Oncol 29: 704-706, 2012.

36. Nabili V, Tan JW, Bhuta S, Sercarz JA and Head CS: Salivary duct carcinoma: A clinical and histologic review with implications for trastuzumab therapy. Head Neck 29: 907-912, 2007.

37. Prat A, Parera M, Reyes V, Peralta S, Cedrés S, Andreu J, Huguet $\mathrm{P}$ and del Campo JM: Successful treatment of pulmonary metastatic salivary ductal carcinoma with trastuzumab-based therapy. Head Neck 30: 680-683, 2008.

38. Ettl T, Stiegler C, Zeitler K, Agaimy A, Zenk J, Reichert TE, Gosau M, Kühnel T, Brockhoff G and Schwarz S: EGFR, HER2, survivin, and loss of pSTAT3 characterize high-grade malignancy in salivary gland cancer with impact on prognosis. Hum Pathol 43: 921-931, 2012.

39. Ito FA, Ito $\mathrm{K}$, Coletta $\mathrm{RD}$, Graner $\mathrm{E}$, de Almeida OP and Lopes MA: Salivary gland tumors: Immunohistochemical study of EGF, EGFR, ErbB-2, FAS and Ki-67. Anal Quant Cytol Histol 31: 280-287, 2009. 